\title{
PRECAUTIONS AGAINST IGNITION OF FLAMMABLE ANAESTHETICS*
}

\section{ELECTROSTATIC HAZARDS IN THE OPERATING THEATRE}

\author{
P. J. SEREDa
}

\section{ELECTRICAL HAZARDS IN THE OPERATING THEATRE}

\author{
A. R. Morse $\nmid$
}

\section{Electrostatic Hazards in the Operating Theatre}

IT MAY SEEM paradoxical to talk about the risks of fire and explosion of anaesthetic gases which occur at a statistical frequency of only one in 80,000 to 100,000 anaesthesias, in comparison with the risks of surgery, which I am sure are many times higher. However, numbers don't always reveal a true picture of the importance of a hazard. We all feel more disturbed about the loss of a neighbour's child due to a car accident which might have been avoided with a little more care than we feel about the loss of many other children due to various diseases about which we can do nothing. These papers are about this "little more care" with regard to the hazard of fire and explosion of anaesthetic gases. Co-operation of engineers, and others who are interested in operating room safety, with members of the medical profession is necessary if the goal of safety is to be attained. We feel the goal is attainable.

For some time many dedicated people have given of their time and effort to study this problem, and the detailed safety codes such as the U.S. NFPA Bulletin 56 are a testimony to this endeavour. It is distressing to realize that even now there are some within the medical profession who show a certain feeling of cold reserve or disinterest towards these safety regulations, as if some external agency was attempting to impose its will and restrict the freedom of the surgeon and the anaesthetist to do a good job in a way they should know best. This is just human nature, but I assure you that those of us who write these codes at the request of, and with the help of, the medical profession have only one motive and that is to save the lives of those who might otherwise die needlessly.

The most important step to safety is the understanding or awareness of the hazard. This is a matter of education. There is no doubt that all of you are aware that open flames, arcs and sparks from non-explosion-proof electrical equipment and faulty wiring, incandescent lamps, hot surfaces of any description, endo-

\footnotetext{
-Delivered to the Section on Anaesthesia of the Ontario Medical Association, Annual Fall Meeting, October 19-20, 1962, at the National Defence Medical Center, Ottawa, Canada.

†Division of Building Research, National Research Council, Ottawa, Canada.

$\ddagger$ Radio and Electrical Engineering Division, National Research Council, Ottawa, Canada.
} 
scopes, high-frequency cauteries, and a glowing cigarette will serve as the source of ignition for mixtures of flammable anaesthetics. However, there is one source of ignition, namely the electrostatic spark, which is not too well understood. This source of ignition is very important since it contributes to the majority of explosions.

What is "static"? We experience its manifestations when we see a bolt of lightning and also when we remove our nylon shirt in the dark. We even hear the noise as in the case of thunder and the crackle of the synthetic fabric. What we see and hear is the spark caused by the sudden discharge of accumlulated electric charges. Any two surfaces brought into intimate contact with one another will assume a condition where the numbers of electrons and ions are not equally distributed between the two surfaces. If the surfaces are both good conductors, then, as the surfaces are separated, the equal distribution of electrons and ions will be achieved on each surface before the last contact bridge is broken. But if even one surface is an insulator then, during separation, the equalization of charges is not possible because the mobility of the electrons is greatly impeded. The time necessary for discharge to take place is dependent upon the resistance and capacitance of the system, and this is countered by the rate of separation of the surfaces.

Many people are of the opinion that both contacting surfaces must be insulators for electrostatic charges to be generated. This is not true. I have here a nickel plate with a square of nylon on it, and a nickel roller. When I pass the roller over the nylon, then drop the nylon into this metal can (or "Faraday Cage," as it is called), you can see the charge that is generated by noting the reading on the electrostatic voltmeter.

Now I wish to show you the effect of induced charging; this demonstrates how a charge existing on a single item may cause many sparks $/$ and still retain its original charge (see Fig. 1). To do this, I close the shorting switch, and the

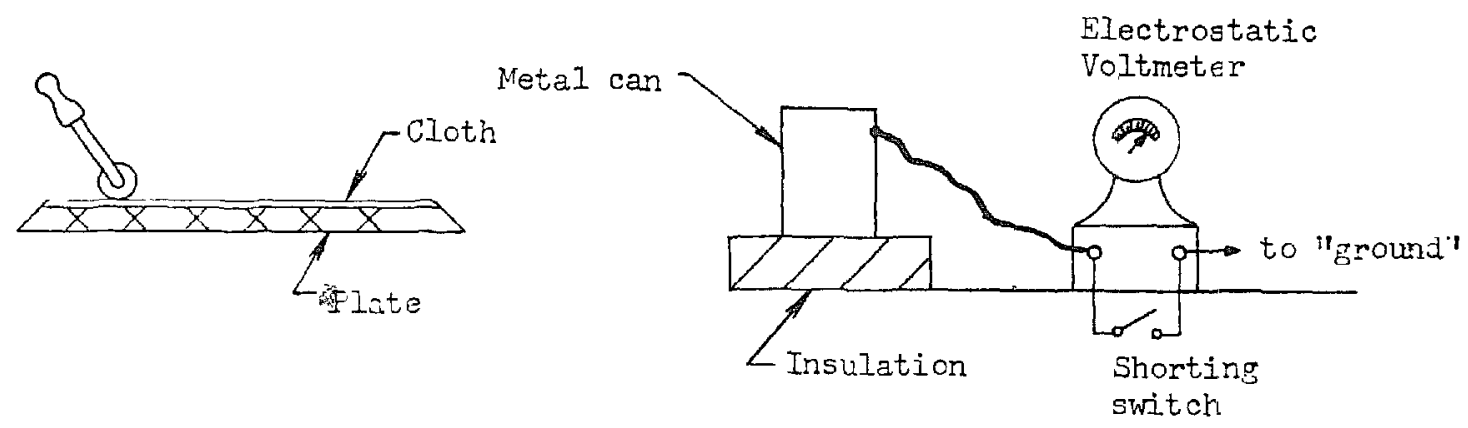

Figure 1.

meter needle swings to zero. What actually happens is that charges move from ground to the can to cause a local neutralization of the charges of opposite polarity on the nylon in the can. Note that these neutralizing charges cannot flow from the can onto the cloth, because the cloth is an insulator. Now if I open the switch you will note that the meter still reads zero, because the system was in equilibrium after the previous step. But as I now remove the cloth with the 
switch still open, we see that the voltmeter again moves to a full-scale reading. This is because the charge that was induced on the can, by the charged cloth, is no longer held in equilibrium. It is now free to cause a spark, as I close the switch, as it discharges to ground. And herein lies the very serious hazard of charged frabrics; for, while the fabric, becautse it is an insulator, can release only small charges at a time, this same fabric can charge by induction an ungrounded conductive article, and cause a heavy spark between this article and ground. Note also that, once charged, the fabric can cause this action any number of times without having to be recharged itself, as I can demonstrate by opening the switch and again lowering the fabric into the can without rubbing the fabric again. While this phenomenon may be a litlle cumbersome to explain, I assure you that in practice it can occur very easily as people with charged clothing move about a room.

Thus the first step in eliminating electrostatic sparks is to remove from use all items that will generate charges and replace them with items having a reasonable degree of conductivity so that charges will not be accumulated. For example, all non-conductive rubber should be replaced with conductive rubber, and synthetic fabrics can be replaced by cotton, which does not generate charges if the humidity is above 50 per cent. (Clean cotton at a humidity of $25-35$ per cent will generate charges that are as high as any synthetic material.)

Care in the selection of material for use in the operating room, and maintenance of a relative humidity of 50 per cent or more, are excellent and necessary steps in avoiding the hazard of static electricity. But it is very difficult indeed to avoid all generation of charges, and for this reason a second line of defence is required. This can be provided most effectively by achieving electrical intercoupling between all items and persons by the provision of static conductive flooring and by having all persons and items make electrical contact with that floor. Static conductive footwear must be worn, for people are especially prone to generate charges because of their activity. It is indeed distressing to find that some hospitals have conductive flooring which they test regularly to ensure conductivity, but in the meantime no one wears conductive footwear, thus nullifying all other efforts.

Static conductive footwear is so important that I should like to discuss it in some detail. Generally, such footwear falls into three categories: conductive shoes, where the sole and heel are made of conductive material and contact the foot (through the moist sock) of the wearer, conductive devices attached to the normal shoe; and the conductive bootie. It seems to me that the most satisfactory solution is the bootie because it already is the standard foot covering in the operating room, and needs only to be modified to ensure some electrical conductivity between the person and the floor. This is achieved by means of a conductive rubber strap which forms part of the bootie and which is used to complete the electrical circuit from the wearer's ankle to the floor. The wearer, of course, has some responsibility-this strap must be tucked in next to the skin and not pulled off as a nuisance item! Whichever type of footwear is used the thing to remember is that it should be tested on the wearer eveny day before the person goes into the operating suite. This is such an important "link" in the safety "chain" that I 
cannot overemphasize it. Recently I had occasion to be in one hospital where I asked to see the shoe tester and I was told by the head nurse that it had been in the corridor at the entrance to the operating room some years ago and that it was removed because it was a nuisance. I mention this because I want to show you that complacency can creep in. The personnel of this hospital probably said: "We've never had any ignitions, so we don't need this." For all they know, the tester may have prevented ignitions-SAFETY is a continuing process.

As with people, equipment must make static conductive contact with the conductive flooring by the use of conductive castors or suitable grounding devices. Drag chains are the poorest of all these devices, not only because they can be wound up on the equipment to "keep them out of the way" (and I have seen this in one hospital recently), but also because the chain can become an insulator when any one of the links becomes covered by dirt or oxide. A chain really needs to be kept tight to assure electrical continuity. However-don't throw the chains away unless you replace them with something better!

Now we have said that all items such as rubber and synthetics should be replaced by static conductive materials. And you will say to me: "But we have done this years ago," but do you periodically test items presently in use to see that in fact they are sufficiently conductive? With use and abuse these items may lose their conductivity (or even be replaced by non-conductive items!) and you may be living in a fool's paradise.

Allow me, therefore, to use the old adage that safety is like a chain, "only as strong as the weakest link." Hence, a true understanding of the electrostatic hazard and its remedies, a constant awareness, and periodic test's and checks are all necessary "links" if you hope to work in a "chain" of conditions free from the hazard of anaesthetic fires or explosions from electrostatic ignition.

\section{Electrical Hazards in the Operating Theatre}

While everyone seems to be at least a little bit careless about electrical shock around the home, it is much more serious if this same small degree of indifference is present in an operating room. Circumstances that so often present only a 'nuisance value in everyday life become matters of great importance under the additional conditions that are present during surgery. For, the same sort of electrical faults that normally cause only annoyance now become a real menace.

There are three distinct serious possibilities. First, faulty circuitry may permit overheating of wires or equipment, or may permit a spark. If this occurs in the presence of flammable anaesthetics, fire or explosion can occur. Second, a member of the staff may receive an electric shock at a moment when an involuntary movement on his part could have secondary consequences. Third, if faulty equipment causes electric currents to pass through the unconscious patient, the patient may be severely burned or may suffer electric shock to the heart. At least two of these possibilities have occurred more than once.' It is not known to us whether shocks to staff have resulted in accidents, but perhaps some of you do know of cases of this nature.

When a hospital is being planned or remodelled, presumably staff who will use the operating suite are consulted regarding requirements. At this stage a tre- 
mendous boost to safety from the three hazards mentioned earlier can be obtained if the staff have a clear idea of what is required. Staff who are called upon for such advice should read the existing codes on "safety during the Use of Anaesthetics," which codes have taken so much labour to prepare, or write the Canadian Standards Association for advice. In this way they will know what to ask the designers for, and why they are asking for it. After all, architects and engineers are like doctors or any other people--they don't always know all the answers, or think of everything. For years and years now much information has been available; yet many hospitals are still being built with protection which is utterly inadequate.

From an electrical point of view, there are three major precautions to be takens The first arises during construction and concerns what is called the permanent installation; the second involves the purchasing of equipment; and the third is concerned with administration, operation, and maintenance.

The permanent installation includes such things as the power supply, panelboards, circuits, switches, outlets, general lighting, the main surgical light, $\mathrm{X}$-ray viewers, clocks, and timers. The general requirement here is that there be one power supply for the permanent installation for each one or two operating rooms, that this supply be ungrounded and isolated from any other supply by means of an isolating transformer, that the whole installation be either explosion-proof or that it be placed above what is considered to be the hazardous area, i.e. placed about five feet from the floor, and that the circuit be continuously monitored to ensure that it remains ungrounded.

(I should perhaps explain what is meant by an ungrounded circuit. An ordinary house circuit has one of the two wires feeding an appliance grounded; in an ungrounded circuit the isolating transformer not only isolates the circuit from the original grounded power source, but also feeds each wire of the circuit with no ground connection on either wire. However, all "dead" or "non-current-carrying" metal is carefully grounded. This system, if monitored to ensure that it stays in good shape, is a wonderful protection against electric shock or accidental electric ignition of anaesthetics.)

To illustrate the necessity for understanding and vigilance, once some blueprints for a new hospital came to our attention just prior to its construction. We found that the operating suite was going to have an isolated ungrounded electrical system-which was admirable-But that the whole suite was going to be supplied by one lonely isolating transformer way down in the basement. Such a system, no matter how carefully installed, would in itself have enough leakage to ground to be hazardous even before any additional electrosurgical equipment was connected to it. Fortunately, this design was changed to permit use of a separate isolated circuit, with its own isolating transformer, for each operating room.

The second major precaution is to purchase electrical equipment, particularly portable equipment, which has been approved for use in the presence of flammable anaesthetics. This may NoT always be possible-but keep demanding it and the manufacturers will produce it, even if they don't now. There are, of course, some devices, such as the radio-frequency cautery, which are virtually impossible to make so that they can pass an approval test. 
It is possible that many of you work in operating rooms that do not have the foregoing protection. If such is the case, then the third precaution is, for you, three times as important. That is, assist the electrical superintendent or electrician to minimize the danger of electrical faults by reporting to him any defects that occur in the electrical system, or any even "harmless" shocks that are received, as these are always a warning signal. This necessity for preventative maintenanee is very important for portable electric equipment, as often this equipment does not receive regular inspection. Such equipment may be, for example, the personal property of a staff member. Not very long ago in Ontario, a piece of portable equipment caused a staff member to receive severe burns when it ignited the anaesthetic. I must sadly confess that I am not surprised that it happened, for I have seen some horrible examples of faulty equipmentsooner or later all the conditions are bound to be right for trouble.

Fortunately, it is becoming more and more possible to use non-flammable anaesthetics-both because better non-flammable anaesthetics are being made available, and because techniques for their use are improving. (I've even read where someone has developed an electric-shock type of anaesthesia-to me, a greenhorn, it sounds like a cross between electric shock therapy and the electric chair. but at least it’s non-flammable!) Nevertheless, every effort should be made to have only approved explosion-proof electrical equipment available, for as you know flammable anaesthetics may have to be used, and on short notice. (This is a point on which advance discussion and/or co-operation between the surgeon and anaesthetist can be very helpful.) Also, and I cannot emphasize this too strongly, avoid LIKE THE PLAGUE doing to a good piece of explosion-proof equipment something that defeats its design-such as using extension cords that have ordinary plugs and sockets.

A problem which is increasing during operative procedures, and which may be of particular interest, is that created by the use of more than one piece of electromedical equipment at one time. Mr. Jack Hopps, of the National Research Council, whom some of you may know for his work with defibrillators, E.E.G.'s and heart-lung equipment, has pointed out that, unless proper care is taken, electric currents may flow from one electric machine, through the unconscious patient, to another machine. In the process the patient may receive severe local burns or electric shock, especially, in some cases, to the heart. As some of these machines are, I believe, monitored by the anaesthetist, he should familiarize himself with their operation and particularly with their proper connection. Ground connections of these mểchines need special care; if care is not taken of both live and ground connections, you won't know that anything is wrong until considerable damage has been done to the patient.

Unfortunately, improved techniques and equipment, which are only devised to give a patient a better chance for a healthy life, bring with them their own problems. They certainly increase the complexity of ensuring safety from both shock and explosion. It is for this reason that codes have to be written, to assist hospital personnel to bring to patients the benefits without the hazards, and it is for this reason that codes sometimes make heavy reading.

No one would suggest for a minute that ${ }^{\circ}$ large city, for example, could get along with the traffic regulations and control of fifty years ago. It is the same in 
the operating room. Some of you probably obtained copies of the proposed Canadian Code for Use of Flammable Anaesthetics which were available at the World Congress of Anaesthetists two years ago. This code was drawn up by a Canadian Standards Association Committee under the Chairmanship of Mr. Gordon Hughes of the Hospital Design Division of the Department of National Health and Welfare. Representatives of all interested parties shared in drawing up this code. Dr. Shapley, for example, was of great help not only in suggestions for safety procedures, but also in enabling other committee members to familiarize themselves with the mysteries of the operating room.

It is our hope that this code will soon be published and available to all. As Moses said when he brought the Ten Commandments down the mountain, "It is a weighty document." But it is weighty not just because of the complexity of the subject, but because the Committee did its best to make it an educational document as well as a code. We feel that on reading this code the hospital staff will really understand not only the hazards which face them-but also the reasons behind every recommendation. I urge you to read this code thoroughly, particularly the Foreword and also Parts I and III, as soon as you are able to obtain a copy. These are the explanatory parts, and the parts which give recommended administrative and operating room procedure. The NFPA pamphlet No. 56, which is the American counterpart, is already available of course, and is also well worth reading.

To sum up:

1. See that the permanent electrical installation is in small isolated ungrounded units, and explosive-proof where required.

2. Demand explosion-proof equipment.

3. Check all equipment carefully and establish a preventative maintenance programme.

4. Use the equipment properly. Don't make "haywire" adaptations and modifications.

5. Establish safe operative procedures beforehand, and be prepared for hitches.

6. Read the Codes and other articles on "Safety during the Use of Flammable Anaesthetics."

7. Always be on your guard-only your alertness can prevent the next rare statistic from occurring in your presence. 